\title{
CONE LENGTH OF THE EXTERIOR JOIN
}

\author{
by HOWARD J. MARCUM
}

(Received 15 January, 1997)

0. Introduction. The cone length $C l(f)$ of a map $f: X \rightarrow Y$ is defined to be the least number of attaching maps possible in a conic (or iterated mapping cone) structure for $f$. Cone length is a homotopy invariant in the sense that if $\varphi: \bar{X} \rightarrow X$ and $\rho: Y \rightarrow \bar{Y}$ are homotopy equivalences then $C l(\rho \circ f \circ \varphi)=C l(f)$. Furthermore $C l(f)$ depends only on the homotopy class of $f$. It was shown by Ganea [8] that the cone length of the map $* \rightarrow X$ coincides with the strong Lusternik-Schnirelmann category of $X$ as a space (see Proposition 1.6 below). Recent work of Cornea ([3]-[6]) is much concerned with cone length and its role in critical point theory. For example, let $f$ be a smooth real valued function on a manifold triad $\left(M ; V_{0}, V_{1}\right)$ with $V_{0} \neq \emptyset$. Under certain conditions, if $f$ has only "reasonable" critical points then it must have at least $C l\left(V_{0} \hookrightarrow M\right)$ of them (see [6]).

In this paper we consider the cone length of the exterior join fibration $p_{\alpha \star \beta}: E(\alpha \star \beta) \rightarrow X \times Y$ for given maps $\alpha: A \rightarrow X$ and $\beta: B \rightarrow Y$ (see [11] or [12] for a definition). The exterior join fibration arises in situations as diverse as Hopf invariants ([7), [15, Part II]) or immersions of real projective spaces. Indeed an example of its occurrence can be quoted as well from Cornea's work mentioned above. In Corollary 2.7 of [6] it is shown that if $M$ and $N$ are differentiable manifolds then the so-called $A$-index of the map $f+g: M \times N \rightarrow \mathbb{R},(f+g)(x, y)=f(x)+g(y)$, is the exterior join of the $A$-indices of given maps $f: M \rightarrow \mathbb{R}$ and $g: N \rightarrow \mathbb{R}$.

As our main result it is shown that the inequality $C l\left(p_{\alpha \star \beta}\right) \leq C l(\alpha) \cdot C l(\beta)$ is valid. Both the equality and the inequality can occur. Our approach in establishing this inequality is to describe specific kinds of attaching maps. We focus especially on slash products (the name is appropriated from the work of Hardie-Porter [9]). Essentially these operations belong to a generalized theory of Whitehead products and this theory is investigated to the extent of giving a cofiber theorem. The main technical lemma used (Lemma 2.1) generalizes [13, Lemma 3.3] and is of independent interest; for instance, the conic structures obtained in [14] may be established by using it.

We work in the category of well-pointed based spaces. All our constructions are made in this category-in particular $\mathcal{M}(f, g)$ denotes the reduced double mapping cylinder on maps $f$ and $g$.

1. Review of cone length. In this section we review some notions related to cone length. Let

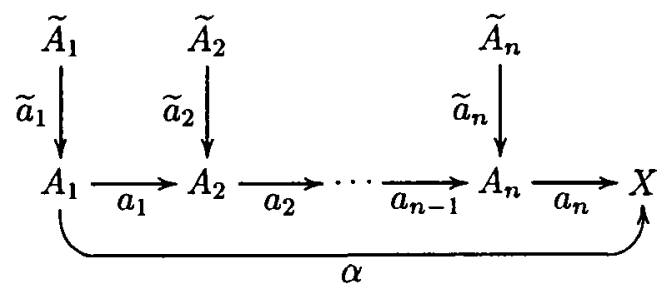

Glasgow Math. J. 40 (1998) 445-461. 
be a conic (or iterated mapping cone) structure for $\alpha: A_{1} \rightarrow X$. By this we mean that there exist homotopies such that each square

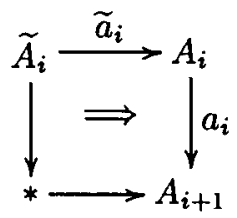

is a homotopy pushout for $i=1, \ldots, n$ (letting $A_{n+1}=X$ ) and that there exists a homotopy $a_{n} \circ \ldots \diamond a_{1} \Rightarrow \alpha$. The minimum number of attaching maps $\tilde{a}_{i}$ possible in a conic structure for $\alpha$ is called the cone length of $\alpha$, denoted $C l(\alpha)$. If no such number exists then $C l(\alpha)=\infty$. If $X$ is a space then we set $C l(X)=C l(* \rightarrow X)$. Thus, for example, $C l(X)=1$ if and only if $X$ has the homotopy type of a suspension space. In particular if $X$ is contractible then $C l(X)=1$.

The following are some properties of cone length. The first property is obvious.

Proposition 1.1. Given $A \stackrel{f}{\rightarrow} X \stackrel{g}{\rightarrow} Z$, then $C l(g \circ f) \leq C l(f)+C l(g)$.

Proposition 1.2. Suppose that the homotopy commutative square

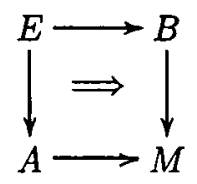

is a homotopy pushout. Then the following inequalities hold.
(a) $C l(B \rightarrow M) \leq C l(A)+1$.
(b) $C l(B \rightarrow M) \leq C l(E \rightarrow A)$.
(c) $C l(M) \leq C l(A)+C l(B)+1$.
(d) $C l(M) \leq C l(E)+\max \{C l(A), C l(B)\}$.

Proof. (a) This is implied by [11, Lemma 3.2].

(b) It is immediate from homotopy pushout properties that any conic structure for $E \rightarrow A$ gives rise to a conic structure for $B \rightarrow M$ which has the same number of attaching maps. The inequality $C l(B \rightarrow M) \leq C l(E \rightarrow A)$ follows.

(c) Clearly $C l(M) \leq C l(B)+C l(B \rightarrow M)$ by Proposition 1.1. But by part (a) we have $C l(B \rightarrow M) \leq C l(A)+1$ and so the inequality asserted in part (c) holds.

(d) By [5, page 703].

COROLLARY 1.3. The inequality $C l(f) \leq C l(X)+1$ is valid for any map $f: A \rightarrow X$.

Proof. Trivially the commutative square

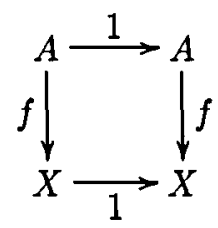


is a homotopy pushout. Hence the inequality which is claimed follows directly by application of Proposition 1.2(a) to this square.

Unless $C l(X)$ is small in value, the inequality in Corollary 1.3 usually provides only a very rough estimate of $C l(f)$. For, typically one can expect $C l(f)$ to be significantly smaller in value than $\mathrm{Cl}(X)$.

Proposition 1.4. Let $\Phi(X)$ denote an integer valued function on spaces having the following properties.

(1) $\Phi(X)=\Phi\left(X^{\prime}\right)$ if $X$ and $X^{\prime}$ are of the same homotopy type.

(2) If $\begin{aligned} C & \rightarrow B \\ * & \rightarrow \downarrow \text { is a homotopy pushout then } \Phi(X) \leq \Phi(B)+1 .\end{aligned}$

Then $\Phi(X) \leq C l(X)+k$ where $k$ is the value of $\Phi$ on contractible spaces.

Proof. Suppose $C l(X)=n$ and that $(1.1)$ is a conic structure for $X$ in which $A_{1} \simeq *$. Then $\Phi\left(A_{2}\right) \leq \Phi\left(A_{1}\right)+1=k+1$ by properties (1) and (2). By iteration

$$
\Phi(X) \leq \Phi\left(A_{n}\right)+1 \leq(k+(n-1))+1=k+n=k+C l(X)
$$

and the proposition is established.

EXAMPLES 1.5 (a) Let cat ${ }^{*}(X)$ denote the pointed Lusternik-Schnirelmann category of a space $X$ as defined in [10, p. 1297] but renormalized to take the value zero on contractible spaces. Then $\operatorname{cat}^{*}(X)$ satisfies properties (1) and (2) with $k=0$. Consequently $\operatorname{cat}^{*}(X) \leq C l(X)$.

(b) Note that $C l(X)$ itself satisfies properties (1) and (2) with $k=1$. This just yields $C l(X) \leq C l(X)+1$.

(c) Recall [8] that the strong Lusternik-Schnirelmann category of a space $X$ is the least interger $n$ such that there is a CW-complex of the same homotopy type as $X$ that can be covered by $n+1$ self-contractible subcomplexes. We consider the based version, denoted $\operatorname{Cat}^{*}(X)$, in which each subcomplex is required to contain the base point. Then $\mathrm{Cat}^{*}(X)$ satisfies properties (1) and (2) with $k=0$. Hence $\operatorname{Cat}^{*}(X) \leq C l(X)$ by Proposition 1.4. In fact these numerical invariants are usually equal, as we next see.

The following result is due to Ganea [8]; also see Cornea [4]. For completeness we include a proof.

Proposition 1.6 (Ganea). If $X$ is path connected and non-contractible then $C l(X)=\operatorname{Cat}^{*}(X)$.

Proof. In view of Example 1.5(c) only the inequality $C l(X) \leq \operatorname{Cat}^{*}(X)$ need be verified. Now if $X$ doesn't have the homotopy type of a CW complex or is not path connected then $\operatorname{Cat}^{*}(X)=\infty$ and there is nothing to prove. Hence assume that $\operatorname{Cat}^{*}(X)=n<\infty$. Since $X$ is non-contractible, $n \geq 1$. Let $Y$ be a CW complex of the same homotopy type as $X$ and let $\left\{U_{i}\right\}_{i=0}^{n}$ be a covering of $Y$ by $n+1$ self-contractible subcomplexes, each containing the base point. Set $Y_{i}=U_{0} \cup \ldots \cup U_{i}$ for $i=0, \ldots n, ; Y_{i}$ is a subcomplex of $Y$. Then $Y_{0}=U_{0} \simeq *, Y_{n}=X$ and we claim that 


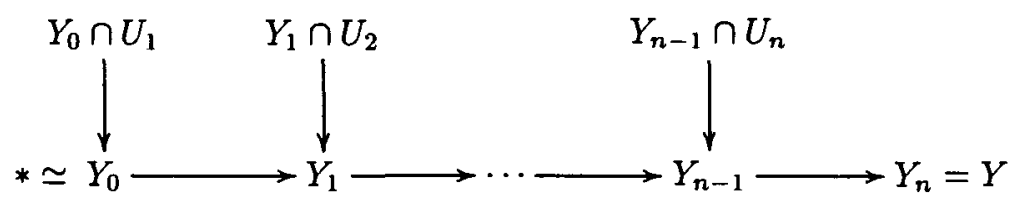

is a conic structure for $* \rightarrow Y$. To see this, note that each square

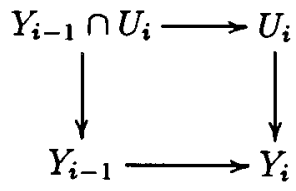

is a topological pushout. This square is also a homotopy pushout since the map $Y_{i-1} \cap U_{i} \rightarrow Y_{i-1}$ is necessarily a cofibration (for $U_{i}$ and $Y_{i-1}$ are subcomplexes). Since $U_{i} \simeq *$ it follows that $Y_{i-1} \cap U_{i} \rightarrow Y_{i-1}$ is an attaching map for $Y_{i-1} \rightarrow Y_{i}$. Because there are $n$ attaching maps we conclude that $C l(X)=C l(Y) \leq n=\operatorname{Cat}^{*}(X)$.

2. A fundamental lemma on certain homotopy colimits. In the next lemma we present an extension of [13, Lemma 3.3]. Also Nomura-Nagase [17] have given a result which may be viewed as a generalization of [13, Lemma 3.3]. Their result is quoted below as Proposition 2.5. Our Lemma 2.1 is the more general result but a relation between the two is indicated in Example 2.4.

LEMMA 2.1. Let the diagram below be given.

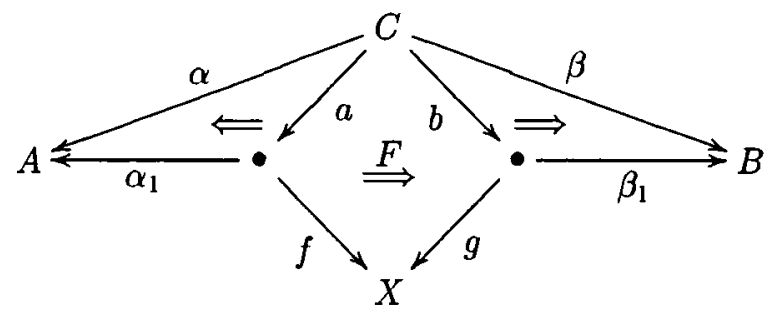

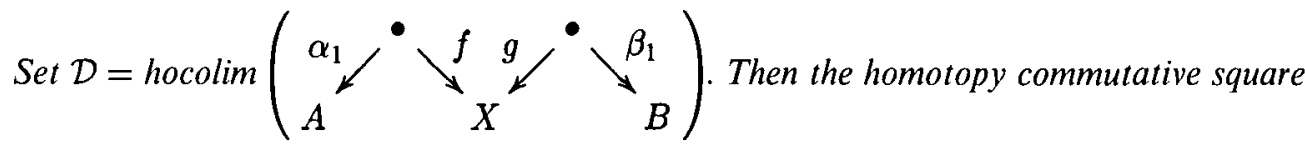

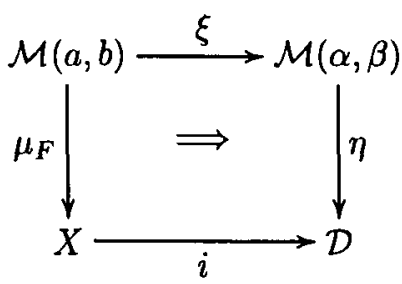

is a homotopy pushout. Note that if $F$ is itself a homotopy pushout then the maps $\mu_{F}$ and $\eta$ are homotopy equivalences. 
Proof. Observe that a homotopy commutative cube

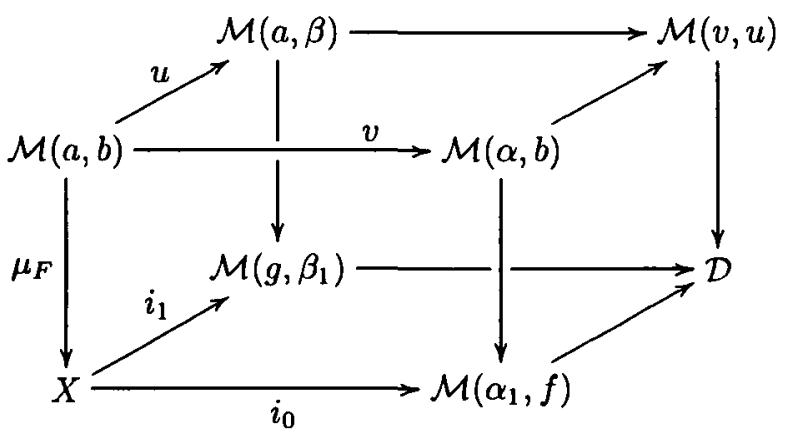

may be formed in which by construction the bottom and top squares are homotopy pushouts. Now by two applications of [13, Lemma 3.3] the front and left squares are seen to be homotopy pushouts. By results of Mather [16] it follows at once that the rear and right squares are also homotopy pushouts. Next we claim that the homotopy commutative square in the statement of the lemma is just the composite in this cube of the front and right squares (or equivalently the left and rear squares). Our claim will be established if we show that $\mathcal{M}(v, u)$ may be identified with $\mathcal{M}(\alpha, \beta)$. Consider the following $3 \times 3$ situation.

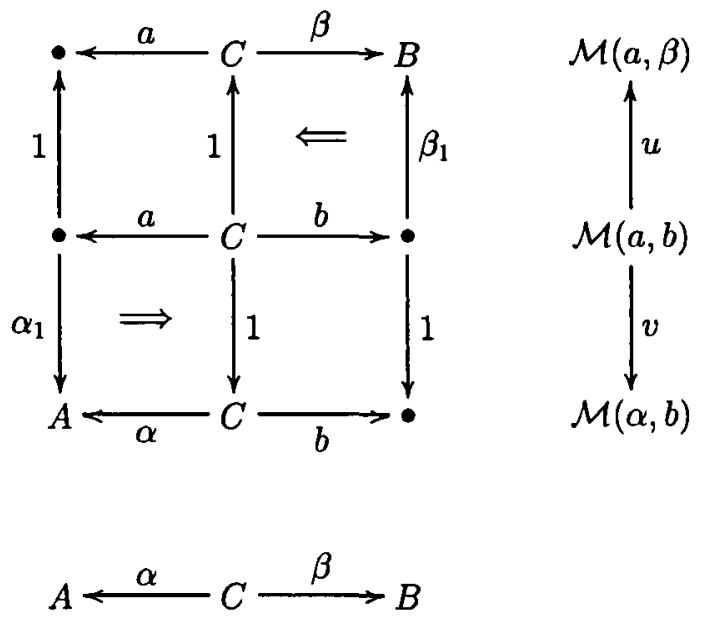

We proceed to take double mapping cylinders both horizontally and vertically as indicated, making use of the identification $\mathcal{M}\left(\alpha_{1}, 1\right) \simeq A, \mathcal{M}\left(1, \beta_{1}\right) \simeq B$ and $\mathcal{M}\left(1_{C}, 1_{C}\right) \simeq C$. An application of [12, Lemma 4.2] then yields the result $\mathcal{M}(\nu, u) \simeq \mathcal{M}(\alpha, \beta)$, as claimed.

That Lemma 2.1 is indeed a generalization of [13, Lemma 3.3] follows from the next proposition.

Proposition 2.2 In Lemma 2.1 we have the following properties.

(a) If $\alpha_{1}$ is an identity map then $\mathcal{D} \simeq \mathcal{M}\left(g, \beta_{1}\right)$.

(b) If $\beta_{1}$ is an identity map then $\mathcal{D} \simeq \mathcal{M}\left(\alpha_{1}, f\right)$. 
Proof. These identifications follow immediately since $\mathcal{D}$ is obtained by taking successive homotopy pushouts.

EXAMPLE 2.3. We consider the diagram

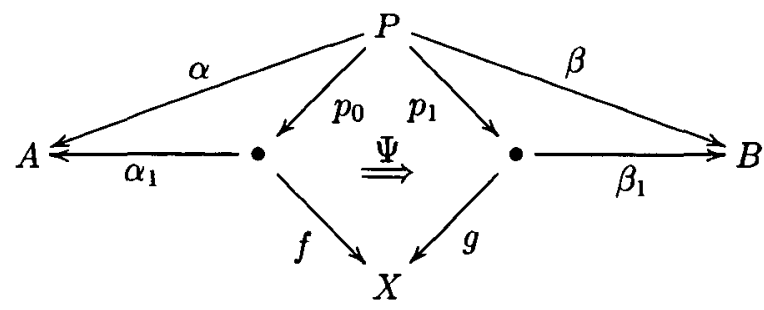

in which $P$ is taken to be just the homotopy pullback of $f$ and $g$ and $\alpha=\alpha_{1} \circ p_{0}, \beta=\beta_{1} \circ p_{1}$. Lemma 2.1 then yields the square

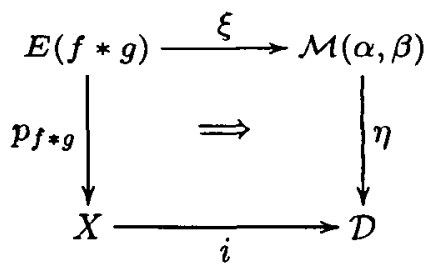

as a homotopy pushout. Here $f * g$ denotes the fiberwise join of $f$ and $g$ (see $\S 2$ of [15, Part I] for a definition of $f * g$ ). It is to be emphasized that $\mathcal{D}$ is an arbitrary homotopy colimit of the form indicated.

EXAMPLE 2.4. Lemma 2.1 may be applied in the context of an arbitrary cube. Let the homotopy commutative cube below, denoted $\mathcal{Q}$, be given.

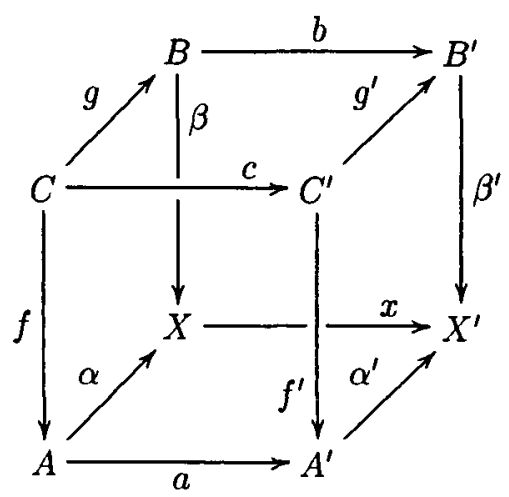

Suppose $F: \alpha \circ f \Rightarrow \beta \circ g$ and $F^{\prime}: \alpha^{\prime} \circ f^{\prime} \Rightarrow \beta^{\prime} \circ g^{\prime}$ and let

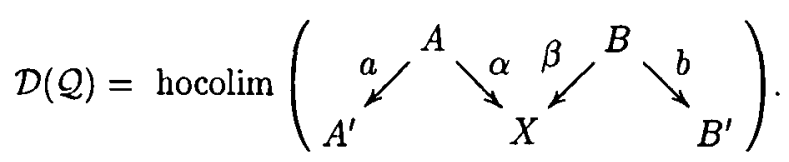


We may then form the homotopy commutative diagram

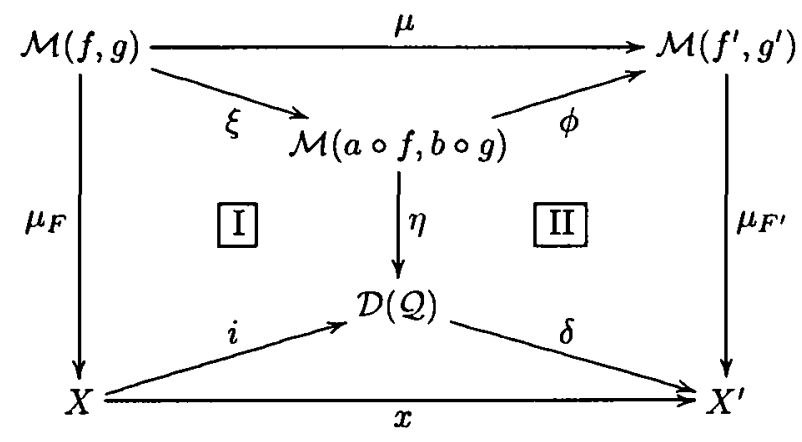

in which the map $\delta$ is induced by homotopy commutativity of the cube. It follows by Lemma 2.1 that square $[$ in this diagram is a homotopy pushout. Furthermore we observe that if in the original cube $c: C \rightarrow C^{\prime}$ is an identity map then $\phi$ is a homotopy equivalence.

Proposition 2.5 ([17, Theorem 1.8]). In the homotopy commutative cube $\mathcal{Q}$ above suppose that the top and bottom squares are homotopy pushouts. Then the induced square

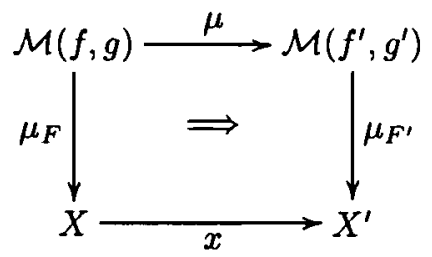

is a homotopy pushout.

Corollary 2.6. Square III in the diagram of Example 2.4 above is a homotopy pushout if the top and bottom squares of $\mathcal{Q}$ are homotopy pushouts.

3. The exterior join case. Let homotopy fractorizations of maps $\alpha$ and $\beta$ be given as follows.

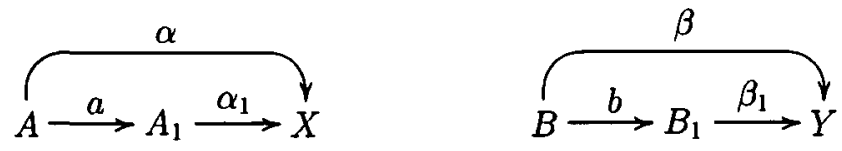

We suppose that specific homotopies $F: \alpha_{1} \circ a \Rightarrow \alpha$ and $G: \beta_{1} \circ b \Rightarrow \beta$ have been selected. Now we define

$$
\mathcal{D}=\mathcal{D}\left(A \stackrel{a}{\rightarrow} A_{1} \stackrel{\alpha_{1}}{\rightarrow} X ; B \stackrel{b}{\rightarrow} B_{1} \stackrel{\beta_{1}}{\rightarrow} Y\right)
$$

to be the homotopy colimit of the diagram:

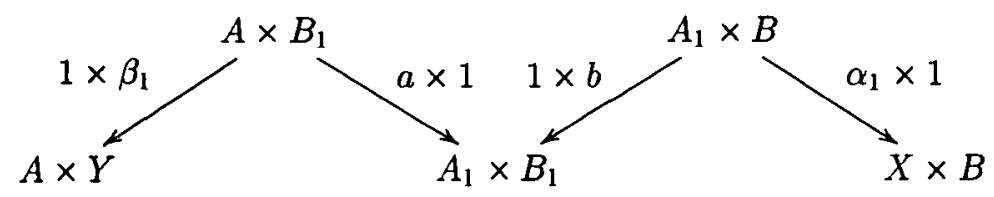


Consider the homotopy commutative cube below.

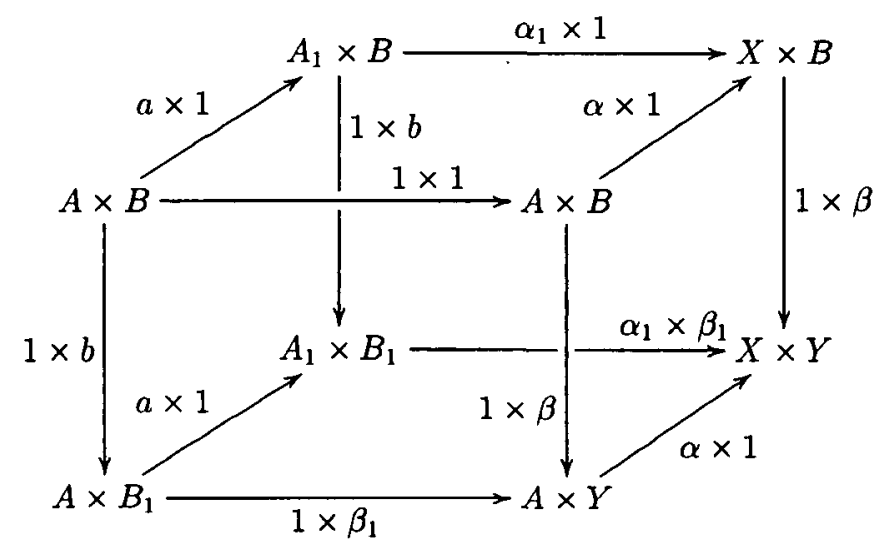

Applying Lemma 2.1 in the context of Example 2.4 and taking note of the presence of the identity map $1 \times 1: A \times B \rightarrow A \times B$ in the cube (which implies that $\phi: \mathcal{M}\left(1 \times \beta_{1} \circ b\right.$, $\left.\alpha_{1} \circ a \times 1\right) \rightarrow E(\alpha \star \beta)$ is a homotopy equivalence) we obtain a homotopy commutative diagram

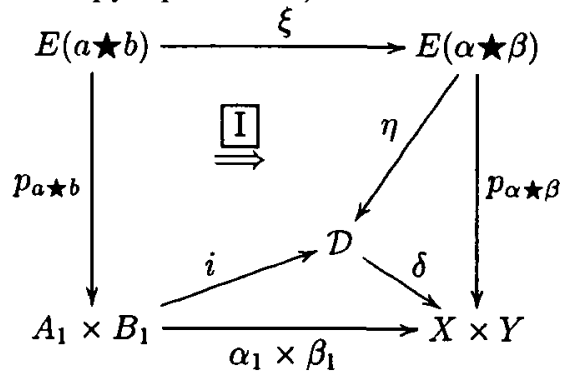

in which square $[\mathrm{I}$ is a homotopy pushout, with induced map $\delta: \mathcal{D} \rightarrow X \times Y$.

Proposition 3.1. The identifications

$$
\begin{aligned}
& \mathcal{D}\left(A_{1} \stackrel{1}{\rightarrow} A_{1} \stackrel{\alpha_{1}}{\rightarrow} X ; B_{1} \stackrel{1}{\rightarrow} B_{1} \stackrel{\beta_{1}}{\rightarrow} Y\right)=E\left(\alpha_{1} \star \beta_{1}\right) \\
& \mathcal{D}\left(A \stackrel{a}{\rightarrow} A_{1} \stackrel{\alpha_{1}}{\rightarrow} X ; B \stackrel{b}{\rightarrow} Y_{1} \stackrel{1}{\rightarrow} Y_{1}\right)=E\left(\alpha_{1} \star b\right) \\
& \mathcal{D}\left(A \stackrel{a}{\rightarrow} A_{1} \stackrel{1}{\rightarrow} A_{1} ; B \stackrel{b}{\rightarrow} B_{1} \stackrel{\beta_{1}}{\rightarrow} Y\right)=E\left(a \star \beta_{1}\right)
\end{aligned}
$$

are valid and each of the squares
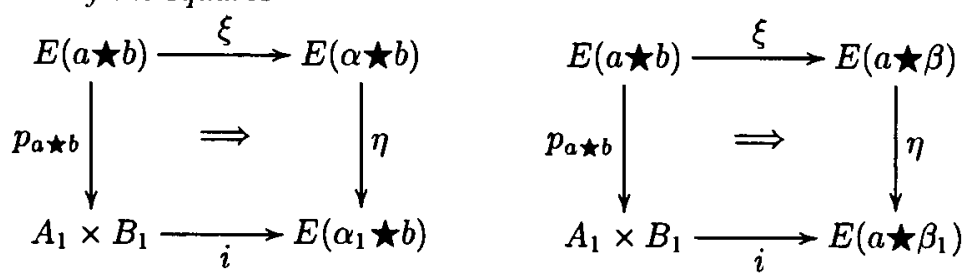

is a homotopy pushout. 
It is interesting to observe that if the spaces $A_{1}$ and $B_{1}$ are each one point spaces then the above squares become cofiber sequences. In fact there is some analogy here to be noted with the cofiber sequence of a triple.

4. Whitehead products via the exterior join functor. In this section we give a presentation of Whitehead products from the point of view of the exterior join functor. Actually our definition of the Whitehead product is more general than the classical one and is somewhat related to the star product approach of Blakers-Massey [2].

Let the following data be specified:
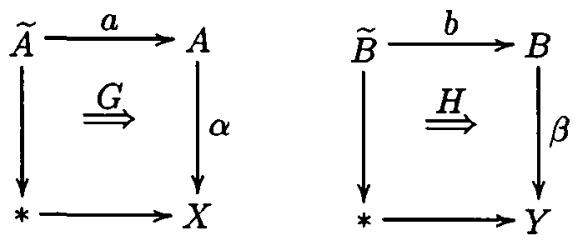

$$
U: E(\alpha \star \beta) \rightarrow Z
$$

Here the homotopies $G$ and $H$ need only be given up to track equivalence and are assumed to be neither homotopy pushouts nor homotopy pullbacks. However we refer to data (4.1) as constituting the cofiber case if indeed $G$ and $H$ are assumed to be homotopy pushouts. Now the two squares in (4.1) determine unique classes $\sigma_{G}: \widetilde{A} \rightarrow F_{\alpha}$ and $\sigma_{H}: \widetilde{B} \rightarrow F_{\beta}$ satisfying $\ell_{\alpha} \circ \sigma_{G}=a$ and $\ell_{\beta} \circ \sigma_{H}=b$ respectively. Then by application of the exterior join functor we obtain a map $w: \widetilde{A} * \widetilde{B} \rightarrow E(\alpha \star \beta)$ and the following homotopy commutative diagram in which the vertical composites are fibration sequences:

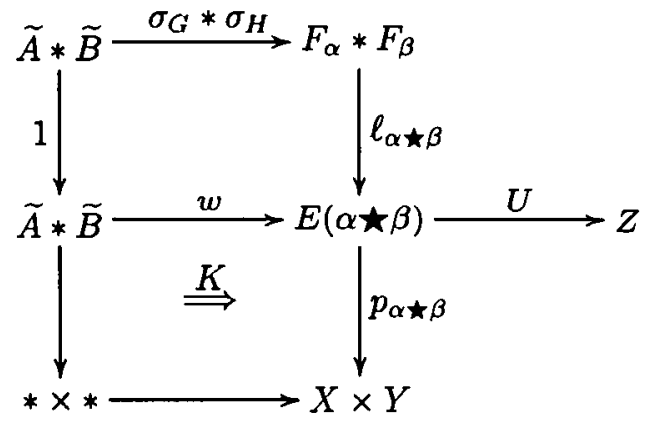

An explicit formula for a representative of $w$ may be taken to be

$$
w[u, v, t]=\left\{\begin{array}{cl}
(a(u), H(v, 4 t)) & , \text { if } 0 \leq t \leq \frac{1}{4} \\
{\left[a(u), b(v), \frac{4 t-1}{2}\right]} & , \text { if } \frac{1}{4} \leq t \leq \frac{3}{4} \\
(G(u, 4-4 t), b(v)) & , \text { if } \frac{3}{4} \leq t \leq 1
\end{array}\right.
$$

for $u \in \widetilde{A}, v \in \widetilde{B}$.

Definition 4.1. In the context of data (4.1) we will refer to $w$ above as the Whitehead product map associated to the squares $G$ and $H$. Also we set $W P\left(U ; \sigma_{G}, \sigma_{H}\right)=$ 
$U \circ w: \widetilde{A} * \widetilde{B} \rightarrow Z$ and call this composite the Whitehead product class corresponding to the data (4.1). Additionally we will say that the map $U: E(\alpha \star \beta) \rightarrow Z$ is $(\alpha \star \beta)$-projective if there exists a map $\gamma: X \times Y \rightarrow Z$ such that $\gamma \circ p_{\alpha \star \beta} \simeq U$.

From diagram (4.2) it is clear that $W P\left(U ; \sigma_{G}, \sigma_{H}\right)=0$ whenever the map $U$ is $(\alpha \star \beta)$ projective. The converse statement is true in the cofiber case, namely:

Proposition 4.2. In the cofiber case of (4.1) the square

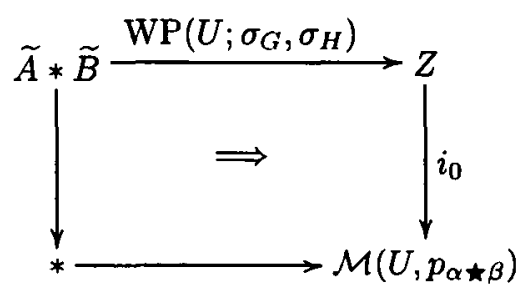

is a homotopy pushout and consequently $W P\left(U ; \sigma_{G}, \sigma_{H}\right)=0$ if and only if $U$ is $(\alpha \star \beta)$-projective.

Proof. Because the exterior join functor preserves homotopy pushouts, the square in (4.2) containing $K$ must be a homotopy pushout when we are in the cofiber case. The proposition follows.

In fact, by using the $\mathcal{D}$-construction, a precise description (covering all cases, not just the cofiber case) of the mapping cone of $w$ in (4.2) may be given, as follows.

THEOREM 4.3. (The Cofiber Theorem for the Whitehead product map). For the data (4.1) consider the homotopy factorizations
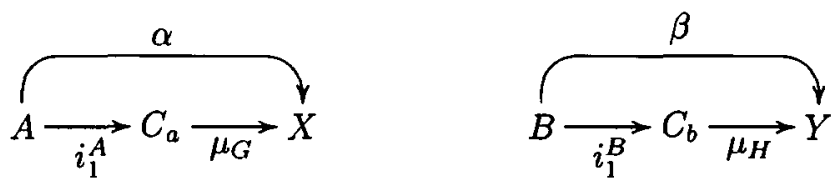

where $\mu_{G}$ and $\mu_{H}$ are the respective maps induced on mapping cones by the homotopies $G$ and $H$. Here $i_{1}^{A}: A \rightarrow C_{a}$ indicates inclusion in the mapping cone at parameter $t=1$ (and similarly for $\left.i_{1}^{B}\right)$. Set

$$
\mathcal{D}=\mathcal{D}\left(A \stackrel{i_{1}}{\longrightarrow} C_{a} \stackrel{\mu_{G}}{\longrightarrow} X ; B \stackrel{i_{1}}{\longrightarrow} C_{b} \stackrel{\mu_{H}}{\longrightarrow} Y\right)
$$

Then the square

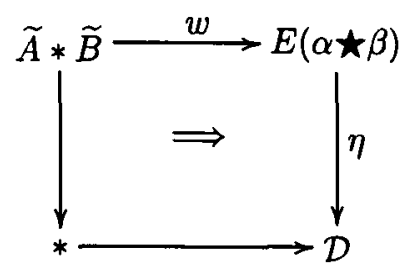

is a homotopy pushout. 
Proof. Under the exterior join functor the composite squares
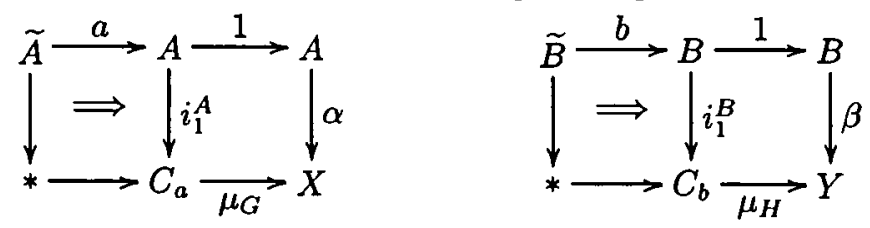

induce the homotopy commutative diagram below.

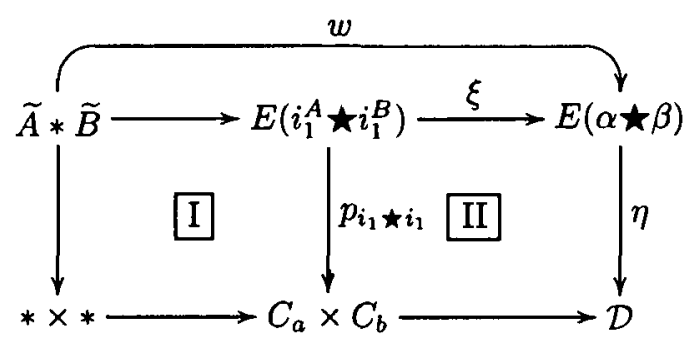

Being an instance of the cofiber case, square $\square$ will be a homotopy pushout. And by (3.2) square II is a homotopy pushout. Thus the composite square is a homotopy pushout as claimed.

EXAMPLE 4.4. We consider the above results in the setting of the ordinary Whitehead product. That is, we suppose given the data

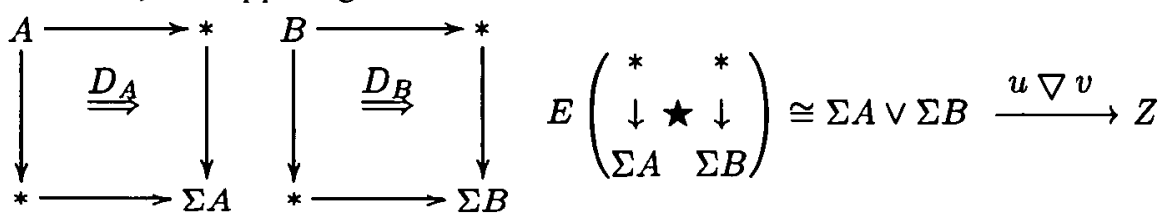

in which $D_{A}$ and $D_{B}$ denote the defining homotopies for the respective suspensions. Note that $\sigma_{D_{A}}=\eta_{A}: A \rightarrow \Omega \Sigma A$ and $\sigma_{D_{B}}=\eta_{B}: B \rightarrow \Omega \Sigma B$ where $\eta_{A}$ and $\eta_{B}$ are the canonical maps.

In this case a check of the definitions shows that the composite arrow

$$
A * B \stackrel{w}{\longrightarrow} E\left(\begin{array}{ccc}
* & & * \\
\downarrow & \star & \downarrow \\
\Sigma A & & \Sigma B
\end{array}\right)=\Sigma B \vee \Sigma A \stackrel{T}{\longrightarrow} \Sigma A \vee \Sigma B
$$

may be identified with the composite

$$
A * B \stackrel{W}{\rightarrow} \Sigma A \vee \Sigma B \stackrel{r \vee r}{\rightarrow} \Sigma A \vee \Sigma B
$$

where $W$ is the ordinary Whitehead product map as defined, say, in [7, page 181], $r$ denotes parameter reversal, and $T$ is interchange of factors. It follows therefore from the Cofiber Theorem 4.3 that the square 


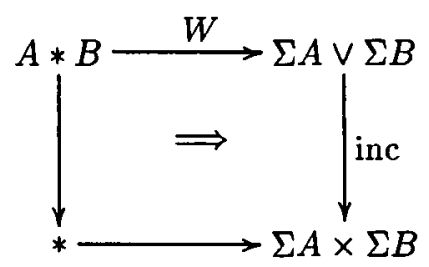

is a homotopy pushout-a classical fact proven in [1, Theorem 4.2]. Furthermore if [, ]; $\pi(\Sigma A, Z) \times \pi(\Sigma B, Z) \rightarrow \pi(A * B, Z)$ denotes the ordinary Whitehead product pairing, then we see that

$$
W P\left((u \nabla v) \circ T ; \eta_{A}, \eta_{B}\right)=[-u,-v]: A * B \rightarrow Z,
$$

for all $u \in \pi(\Sigma A, Z), v \in \pi(\Sigma B, Z)$.

5. The slash product map construction. In this section we give a construction of the slash product map; this construction is viewed as one generalization of the Whitehead product map defined in Definition 4.1. There is both a left and a right version to be considered. These are denoted $\left(\alpha / \alpha_{1}, \beta\right)$ and $\left(\alpha, \beta / \beta_{1}\right)$ respectively. They have the functorial forms indicated below.

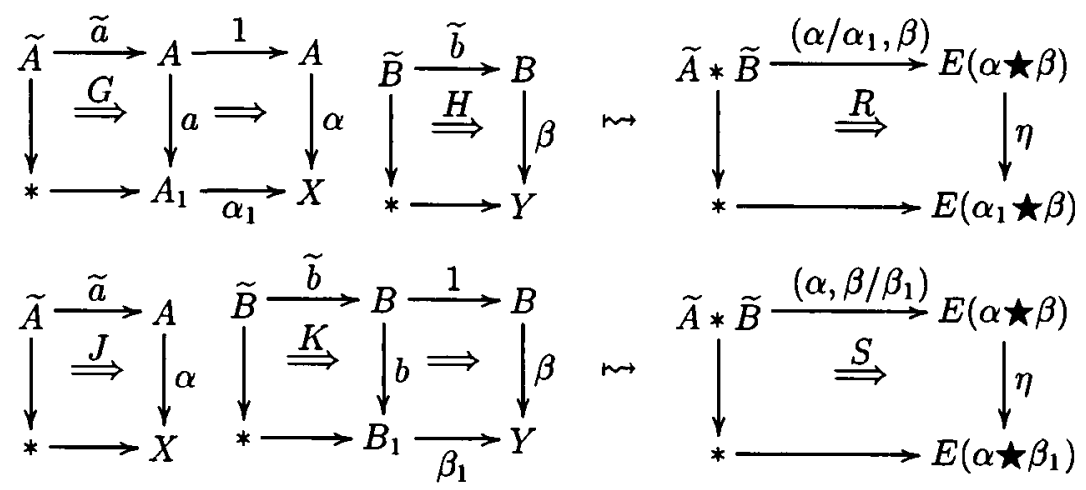

For example, the square $R$ in (5.1) is by definition the following composite square.

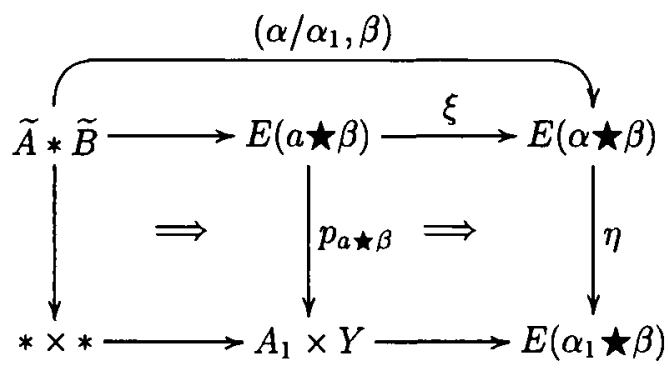

Here the left square is obtained by applying the exterior join functor to the squares $G$ and $H$, while the right square is obtained as in Proposition 3.1 and is always a homotopy pushout. Of course the left square will be a homotopy pushout if $G$ and $H$ are homotopy pushouts (since the exterior join functor preserves homotopy pushouts). These remarks yield part (a) in Proposition 5.1 below. The similar definition and analysis of $\left(\alpha, \beta / \beta_{1}\right)$ is omitted. 
Proposition 5.1 (a) If in (5.1) the squares $G$ and $H$ are homotopy pushouts then so is $R$. (b) If in (5.2) the squares $J$ and $K$ are homotopy pushouts then so is $S$.

REMARK 5.2. Given squares
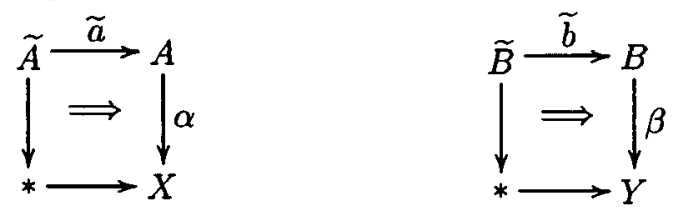

it is clear that the identifications

$$
\left(\alpha / 1_{X}, \beta\right)=w=\left(\alpha, \beta / 1_{Y}\right): \widetilde{A} * \widetilde{B} \rightarrow E(\alpha \star \beta)
$$

are valid. That is, the slash product map construction generalizes the Whitehead product map construction.

Referring to the conic structure (1.1) let $\alpha_{i}: A_{i} \rightarrow X$ denote the composite

$$
A_{i} \stackrel{a_{i}}{\rightarrow} A_{i+1} \stackrel{a_{i+1}}{\rightarrow} \ldots \rightarrow A_{n} \stackrel{a_{n}}{\rightarrow} X .
$$

In particular $\alpha_{n}=a_{n}$. Also let the square

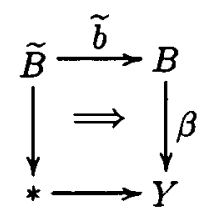

be assumed to be a homotopy pushout. By iterating Proposition 5.1 we thus have:

Proposition 5.3. Under the hypotheses just described, the diagram

$$
\begin{aligned}
& \begin{array}{ccc}
\widetilde{A}_{1} * \widetilde{B} & \widetilde{A}_{2} * \widetilde{B} & \widetilde{A}_{n} * \widetilde{B}
\end{array} \\
& \left(\alpha_{1} / \alpha_{2}, \beta\right) \downarrow \quad\left(\alpha_{2} / \alpha_{3}, \beta\right) \downarrow \quad\left(\alpha_{n} / 1_{X}, \beta\right) \downarrow \\
& \underbrace{E\left(\alpha_{1} \star \beta\right) \longrightarrow E\left(\alpha_{2} \star \beta\right) \longrightarrow}_{p_{\alpha_{1} \star \beta}} \longrightarrow\left(\alpha_{n} \star \beta\right) \underset{p_{\alpha_{n} \star \beta}}{\longrightarrow} X \times Y
\end{aligned}
$$

constitutes a conic structure for $p_{\alpha_{1} \beta \beta}$. Consequently, $C l\left(p_{\alpha_{1} \beta \beta}\right) \leq C l\left(\alpha_{1}\right)$ whenever $C l(\beta)=1$.

In Proposition 5.3 it is to be emphasized that all the attaching maps in the conic structure are slash product maps, with the top attaching map $\left(\alpha_{n} / 1_{X}, \beta\right)$ being a Whitehead product map, by Remark 5.2.

Example 5.4. In this example we examine the cone length of the projection map $\operatorname{pr}_{Y}: X \times Y \rightarrow Y$. By Corollary 1.3 we have $C l\left(\operatorname{pr}_{Y}\right) \leq C l(Y)+1$. We note that this same estimate can be obtained via Proposition 5.3 even though $\operatorname{pr}_{Y}$ itself is not an exterior join fibration. To see this we refer to the diagram 


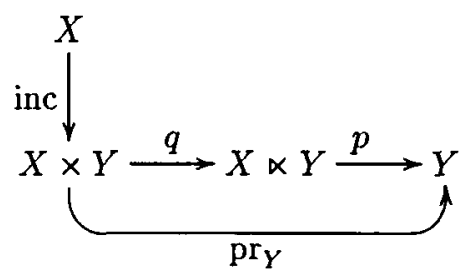

where by definition the half-smash $X \propto Y$ is the cofiber of the inclusion of $X=X \times *$ into $X \times Y$. By Proposition 1.1 we have $C l\left(\operatorname{pr}_{Y}\right) \leq C l(p)+C l(q)$. Plainly $C l(q)=1$. Next we note that the map $p$ is an exterior join fibration since the identification

$$
E\left(\begin{array}{lll}
X & & * \\
\downarrow & \star & \downarrow \\
* & & Y
\end{array}\right)=\mathcal{M}(X \times Y \stackrel{1 \times 0}{\longleftarrow} X \times * \stackrel{0 \times 1}{\longrightarrow} * \times *) \simeq X \ltimes Y
$$

is valid. Furthermore Proposition 5.3 is applicable because $C l(X \rightarrow *)=1$; hence $C l(p) \leq C l(Y)$. In consequence the inequality

$$
C l\left(\operatorname{pr}_{Y}: X \times Y \rightarrow Y\right) \leq C l(Y)+1
$$

is again obtained.

REMARK 5.5. As mentioned in the introduction the name "slash product" is appropriated from Hardie-Porter [9]. The relevant example there concerns the map

$$
\left(\iota_{p} \times \iota_{q}\right) / e_{p+1}: S^{p+q}=S^{p} * S^{q-1} \rightarrow E\left(\begin{array}{ccc}
S^{p} & & * \\
\downarrow & \star & \downarrow \\
* & & S^{q}
\end{array}\right)=\left(S^{p} \times S^{q}\right) \cup\left(E^{p+1} \times *\right)
$$

(see [9, Definition 1]) which from the viewpoint adopted in this article would be called a Whitehead product rather than a slash product.

6. The cone length of $p_{\alpha \star \beta}$. This section is devoted to proving the following theorem.

TheOrem 6.1. For maps $A \rightarrow X$ and $\beta: B \rightarrow Y$ the inequality

$$
C l\left(p_{\alpha \star \beta}\right) \leq C l(\alpha) \cdot C l(\beta)
$$

is valid.

Suppose $C l(\alpha)=n \geq 1$ and $C l(\beta)=k \geq 1$. Let conic structures

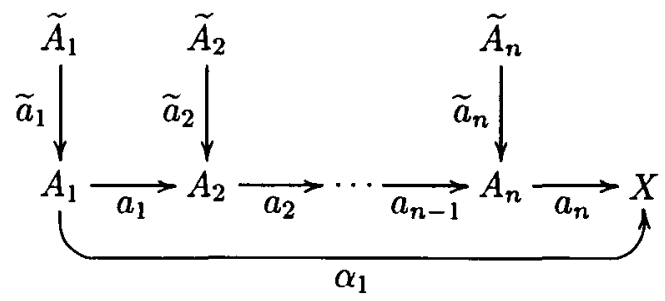


and

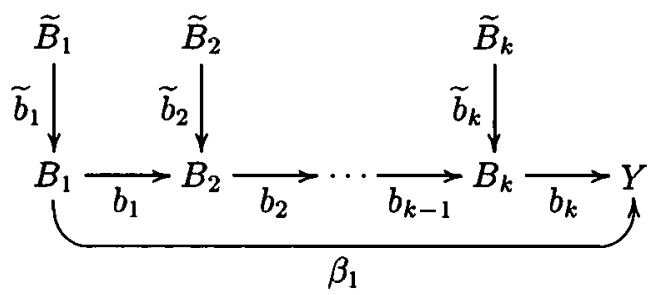

be given where $A_{1}=A, \alpha_{1}=\alpha, B_{1}=B$ and $\beta_{1}=\beta$. As usual we let $\alpha_{i}: A_{i} \rightarrow X$ and $\beta_{j}: B_{j} \rightarrow Y$ denote the composites

$$
\begin{gathered}
A_{i} \stackrel{a_{i}}{\rightarrow} A_{i+1} \stackrel{a_{i+1}}{\rightarrow} \ldots \rightarrow A_{n} \stackrel{a_{n}}{\rightarrow} X \\
B_{j} \stackrel{b_{j}}{\rightarrow} B_{j+1} \stackrel{b_{j+1}}{\rightarrow} \ldots \rightarrow B_{k} \stackrel{b_{k}}{\rightarrow} Y
\end{gathered}
$$

respectively for $1 \leq i \leq n, 1 \leq j \leq k$. Of course $\alpha_{n}=a_{n}$ and $\beta_{k}=b_{k}$. We consider the factorization

$$
E\left(\alpha_{1} \star \beta_{1}\right) \stackrel{\eta_{1}}{\rightarrow} E\left(\alpha_{2} \star \beta_{1}\right) \stackrel{\eta_{2}}{\rightarrow} \ldots \stackrel{\eta_{n-1}}{\rightarrow} E\left(\alpha_{n} \star \beta_{1}\right) \stackrel{\eta_{n}}{\rightarrow} E\left(1_{X} \star \beta_{1}\right) \simeq X \times Y
$$

of $p_{\alpha_{1} \star \beta_{1}}$; note that this has $C l\left(\alpha_{1}\right)$ factors. Now for each $i=1, \ldots, n$ the square

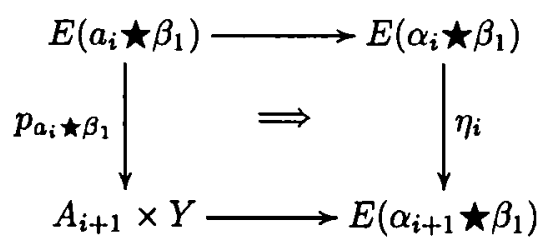

is a homotopy pushout, by Proposition 3.1. That is to say, the above factorization decomposes $p_{\alpha_{1} \star \beta_{1}}$ into iterated double mapping cylinders. Also $C l\left(p_{a_{i} \star \beta_{1}}\right) \leq C l\left(\beta_{1}\right)$ by Proposition 5.3 since $C l\left(a_{i}\right)=1$. Hence by Proposition $1.2(\mathrm{~b})$ we deduce that

$$
C l\left(\eta_{i}: E\left(\alpha_{i} \star \beta_{1}\right) \rightarrow E\left(\alpha_{i+1} \star \beta_{1}\right)\right) \leq C l\left(\beta_{1}\right)
$$

and consequently the inequality $C l\left(p_{\alpha_{1} \star \beta_{1}}\right) \leq C l\left(\alpha_{1}\right) \cdot C l\left(\beta_{1}\right)$ is obtained since as was pointed out above there are $C l\left(\alpha_{1}\right)$ factors in the factorization of $p_{\alpha_{1} \star \beta_{1}}$. This completes the proof of Theorem 6.1.

In Theorem 6.1 one should note that not all the attaching maps are describable as slash product maps. Indeed a sequence of slash product maps does occur in the conic structure but interspersed between any two such are other attaching maps which we have not described explicitly.

7. Concluding remarks. For spaces $X$ and $Y$ let $X \vee Y \rightarrow X \times Y$ be the inclusion of the wedge into the product. It is instructive to look at Theorem 6.1 as applied to this example. 
Such an application is possible since this inclusion may be identified with the exterior join fibration of the maps $* \rightarrow X$ and $* \rightarrow Y$ (cf. [15, Part II]). Hence Theorem 6.1 yields the inequality

$$
C l(X \vee Y \rightarrow X \times Y) \leq C l(X) \cdot C l(Y)
$$

However this inequality is not a sharp result. For example, assume that $X$ and $Y$ are spaces each having cone length $\leq 2$. Then (7.1) gives 4 as an upper bound for the cone length of $X \vee Y \rightarrow X \times Y$. But it is shown in [11, (6.1)] that the inequality $C l(X \vee Y \rightarrow X \times Y) \leq 3$ obtains and indeed appropriate attaching maps are described there.

Finally we note that Corollary 1.3 may be applied to $p_{\alpha \star \beta}: E(\alpha \star \beta) \rightarrow X \times Y$ as a map. This yields the inequality $C l\left(p_{\alpha \star \beta}\right) \leq C l(X \times Y)+1$ and we wish to compare this estimate with that given in Theorem 6.1. We restrict attention to spaces $X$ and $Y$ that are pathconnected and non-contractible. Then by Proposition 1.6 we may write $C l(X \times Y)=$ $\operatorname{Cat}^{*}(X \times Y)$. Now it is a result of Takens [18] that $\operatorname{Cat}^{*}(X \times Y) \leq \operatorname{Cat}^{*}(Y)$ in this case. Hence the inequality

$$
C l\left(p_{\alpha \star \beta}\right) \leq C l(X)+C l(Y)+1
$$

is obtained. We observe that sometimes it is Theorem 6.1 that is the better estimate, sometimes (7.2).

\section{REFERENCES}

1. M. Arkowitz, The generalized Whitehead product, Pacific J. Math. 12 (1962), 7-23. 295-324.

2. A. L. Blakers and W. S. Massey, Products in homotopy theory, Ann. of Math. (2) 58 (1953),

3. O. Corena, Cone-length and Lusternik-Schnirelmann category, Topology 33 (1994), 95-111.

4. O. Corena, Strong LS category equals cone-length, Topology 34 (1995), 377-381.

5. O. Corena, Lusternik-Schnirelmann-categorical sections, Ann. Scient. Éc. Norm. Sup. 28 (1995), 689-704.

6. O. Corena, Cone-decompositions and degenerate critical points, Proc. London Math. Soc. 77 (1998), 437-461.

7. G. Dula and H. J. Marcum, Hopf invariants of the Berstein-Hilton-Ganea kind, Topology Appl. 65 (1995), 179-203. $417-427$

8. T. Ganea, Lusternik-Schnirelmann category and strong category, Illinois J. Math. 11 (1967),

9. K. A. Hardie and G. J. Porter, The slash product homotopy operation, Proc. London Math. Soc. (3) 34 (1977), 505-519.

10. I. M. James, Lusternik-Schnirelmann category, in Handbook of Algebraic Topology (NorthHolland, 1995), 1293-1310.

11. H. J. Marcum, Homotopy decompositions for product spaces in Atas do Décimo Primeiro Colóquio Brasileiro de Matemática, vol. II, (Instituto de Matematica Pura e aplicada (IMPA), Rio de Janeiro, 1978), pp. 665-680.

12. H. J. Marcum, Fibrations over double mapping cylinders, Illinois J. Math. 24 (1980), 344-358.

13. H. J. Marcum, Two results on cofibers, Pacific J. Math. 95 (1981), 133-142.

14. H. J. Marcum, Obstructions for a map to be cyclic, in Algebraic Topology: Oaxtepec 1991, Contemporary Mathematics Series, vol. 146, (American Mathematical Society, 1993), 277-295.

15. H. J. Marcum, Functional properties of the Hopf invariant I, Quaestiones Math. 19 (1996), 537-587; II, preprint.

16. M. Mather, Pull-backs in homotopy theory, Canad. J. Math. 28 (1976), 225-263. 
17. Y. Nomura and T. Nagase, On homotopy commutative squares and cubes, Sci. Rep., Col. Gen. Educ. Osaka Univ. 30 (1982), 91-117.

18. F. Takens, The Lusternik-Schnirelman categories of a product space, Compositio Math. 22 (1970), 175-180.

The Ohio State University at Newark

1179 University Drive, Newark

Онго 43055, USA

E-mail: marcum@math.ohio-state.edu 